\title{
Applications of Soft X-ray Spectromicroscopies to Lithium Ion Battery Analysis
}

\author{
Jian Wang ${ }^{1}$ \\ 1. Canadian Light Source Inc., Saskatoon, Canada \\ * Corresponding author, jian.wang@lightsource.ca
}

Modern battery technologies, particularly lithium ion batteries (LIBs), are being implemented very rapidly in applications from portable consumer electronics to large electric vehicles. Their performance is critically related to the electrode materials and battery fabrication technologies. An in-depth structural and chemical understanding of electrode materials and the interplay among battery components is key to performance enhancement and development of new systems. The soft X-ray spectromicroscopy (SM) beamline at the Canadian Light Source (CLS) [1] consists of two types of X-ray microscopes. There are two bulk sensitive Scanning Transmission X-ray Microscopes (STXM) [Ambient-STXM and the recently commissioned Cryo-STXM], and a surface sensitive X-ray Photoemission Electron Microscope (XPEEM). Both types of microscopes are well suited to the requirements for analysis of LIB materials with respect to characterizations of morphology/structure, identification and quantitative distributions of chemical components in 2D and 3D, molecular orientation, etc., using X-ray Absorption Near Edge Structure (XANES) based spectromicroscopy.

STXM is being used to determine the state of charge (SOC) or oxidation state, charging/discharging mechanisms, and chemical interactions. Fig. 1 gives examples of SOC imaging in a novel $\mathrm{LiMn}_{0.75} \mathrm{Fe}_{0.25} \mathrm{PO}_{4}$ nanorod-graphene hybrid battery material [2]. Fig. 1a shows a mixed charge state of fully delithiated $\mathrm{Fe}^{3+}$ and native $\mathrm{Fe}^{2+}$ in the partially charged electrode. The well resolved individual nanorods $(\sim 100 \mathrm{~nm})$ of pure $\mathrm{Fe}^{3+}$ suggest a domino-cascade delithiation model with the lithium transportation channel [010] along the crosssection of the nanorods and perpendicular to the attached graphene. Furthermore, spatially resolved C K-edge XANES spectroscopy (Fig. 1b) shows there is strong interaction between the nanorods of $\mathrm{Fe}^{3+}$ and the graphene support, as reflected by the significantly enhanced $288 \mathrm{eV}$ feature. This feature can be used to correlate structural and chemical variations with performance differences. Fig. 1c and 1d display the STXM chemical imaging and local spectroscopy of a partially charged $\mathrm{Li}_{2} \mathrm{FeSiO}_{4}$ (LFS) battery material [3]. The STXM derived chemical map (Fig. 1c) shows that the agglomerated LFS nanoparticles experience different degrees of delithiation as denoted by different colors [red is $\mathrm{Fe}^{3+}$, green is $\mathrm{Fe}^{2+}$, and orange is a mix]. The distribution of the orange color indicates that $\mathrm{Li}$ ion diffusion occurs from the LFS/electrolyte interface to
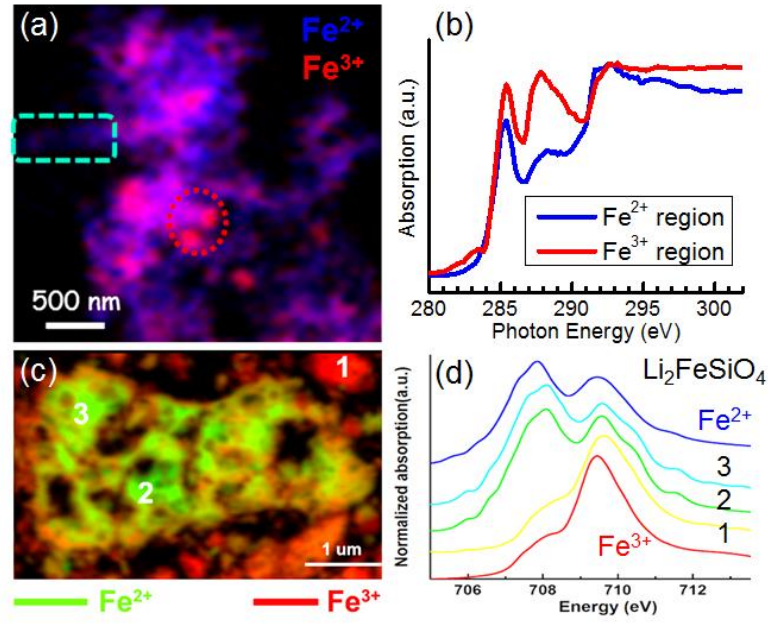

Figure 1. (a) STXM chemical map of the state of charge in the cycled $\mathrm{LiMn}_{0.75} \mathrm{Fe}_{0.25} \mathrm{PO}_{4}$ nanorods-graphene hybrid at the Fe L-edge, blue: $\mathrm{Fe}^{2+}$, red: $\mathrm{Fe}^{3+}$; (b) $\mathrm{C}$ K-edge XANES from the selected regions as outlined in (a), the dashed box for $\mathrm{Fe}^{2+}$ region, and the dashed circle for $\mathrm{Fe}^{3+}$ region (adapted from [2]). (c) STXM chemical map of the delithiated $\mathrm{Li}_{2} \mathrm{FeSiO}_{4}$ electrode at the Fe L-edge, green: $\mathrm{Fe}^{2+}$, red: $\mathrm{Fe}^{3+}$; (d) $\mathrm{Fe}$ L-edge XANES from numbered regions in (c), compared with $\mathrm{Fe}$ reference spectra (adapted from [3]). 
the inside of the LFS particles through phase boundary displacements. Fe L-edge XANES spectra (Fig. 1d), acquired from the local regions labelled 1 to 3 (Fig. 1c), confirmed the variation in the localized chemical states in the material. Specifically, region 1 is $\mathrm{LiFeSiO}_{4}$, and regions 2 and 3 are discharged products, $\mathrm{Li}_{1.5} \mathrm{FeSiO}_{4}$ and $\mathrm{Li}_{2} \mathrm{FeSiO}_{4}$, respectively. The electrochemical process is not able to achieve uniform Li content throughout the LFS electrode, and results from variations in particle size, distributions and electrolyte penetration.

Transmission detection in the soft X-ray energy range is very challenging or even impossible for micrometer size LIB materials and electrode assemblies. To overcome these issues, novel imaging approaches, using energy dispersive X-ray fluorescence (XRF) recorded with a silicon drift detector (SDD) (100 nm sampling depth) (Fig. 2a) and total electron yield (TEY) detection through sample photocurrent (1 nm sampling depth) (Fig. 2b) were implemented at CLS for LIB materials analysis. Fig. 2c shows XRF-STXM imaging of pristine $\mathrm{LiNi}_{0.33} \mathrm{Fe}_{0.33} \mathrm{Mn}_{1.33} \mathrm{O}_{4}$ battery cathode crystals [4]. The (111) and (100) crystalline facets are seen and their chemical composition identified. Several Fe/Ni rich hot spots at the crystal edges and random facet inhomogeneity such as Mn rich (111) facets are observed. Furthermore, the $\mathrm{Mn} \mathrm{L}_{3}$-edge XRF-XANES (Fig. 2d) of those two facets shows the (111) facet is dominated by $\mathrm{Mn}^{4+}$, while the (100) facet has a contribution from $\mathrm{Mn}^{3+}$, as suggested by the enhanced spectral intensity around $642 \mathrm{eV}$ and lower energies. This implies a Ni/Mn disordered phase with oxygen vacancies that are compensated by Mn reduction. Fig. 2e shows the TEY-STXM imaging of a well-shaped single crystal. The TEY-XANES (Fig. 2f), extracted from the crystal, confirms the facet surface (dark regions) is dominated by stoichiometric $\mathrm{Mn}^{4+}$, while there is a $\mathrm{Mn}^{3+} / \mathrm{Mn}^{4+}$ mixture at the bright crystal edges, suggesting a Ni/Mn disordered phase.
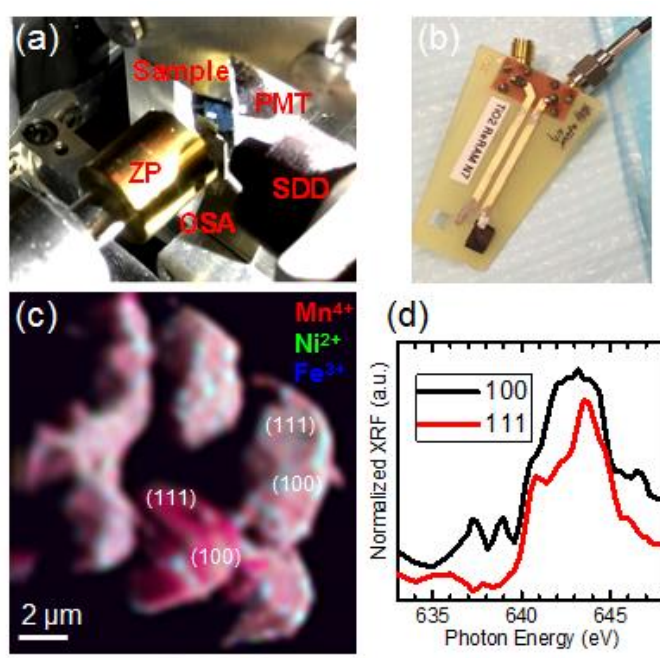

(d)
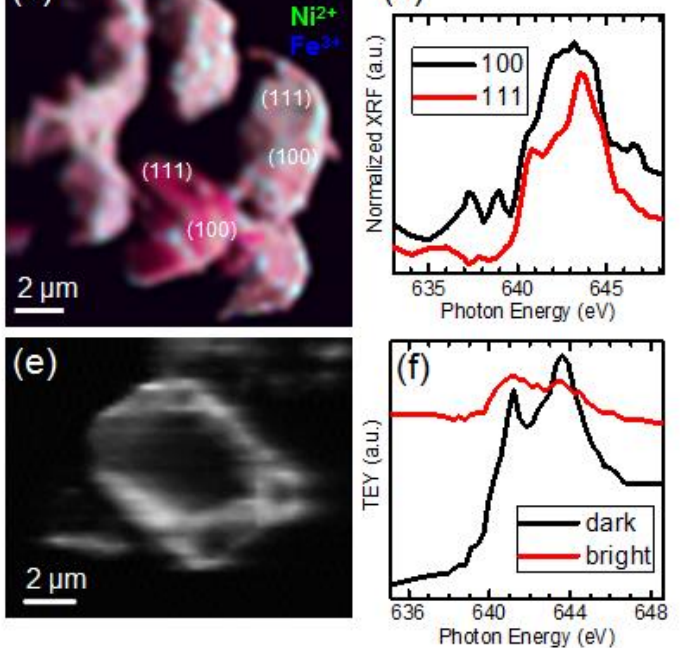

Figure 2. (a) XRF-STXM setup; (b) TEY-STXM sample holder; (c) chemical map of the pristine $\mathrm{LiNi}_{0.33} \mathrm{Fe}_{0.33} \mathrm{Mn}_{1.33} \mathrm{O}_{4} \quad$ (LNFMO) cathode crystals, red: $\mathrm{Mn}^{4+}$, green: $\mathrm{Ni}^{2+}$, blue: $\mathrm{Fe}^{3+}$; (d) $\mathrm{Mn}$ L-edge XRF-XANES from the adjacent (111) and (100) facets; (e) TEY-STXM morphologic imaging of a well-shaped LNFMO single crystal; (f) Mn L-edge TEY-XANES from dark and bright regions on the crystal in (e) (adapted from [4]).

CLS X-PEEM is a commercial instrument from Elmitec, ideal for UHV compatible, flat and conductive sample surface characterization. Despite the many challenges for applying X-PEEM to battery electrodes due to rough and poor vacuum compatible electrode surfaces, direct X-PEEM spectromicroscopy of LIB battery electrodes has been achieved at the CLS [5]. These results probe the important electrode surface and interface information as well as surface conductivity. Fig. 3a and 3b display X-PEEM results of a study of the effect of suberonitrile (SUN) and lithium bis(oxalate)borate (LiBOB) binary additives on the $\mathrm{LiCoO}_{2} / \mathrm{LiPF}_{6}$ interface in a cycled $\mathrm{LiCoO}_{2}$ (LCO) composite electrode. This study provided information 

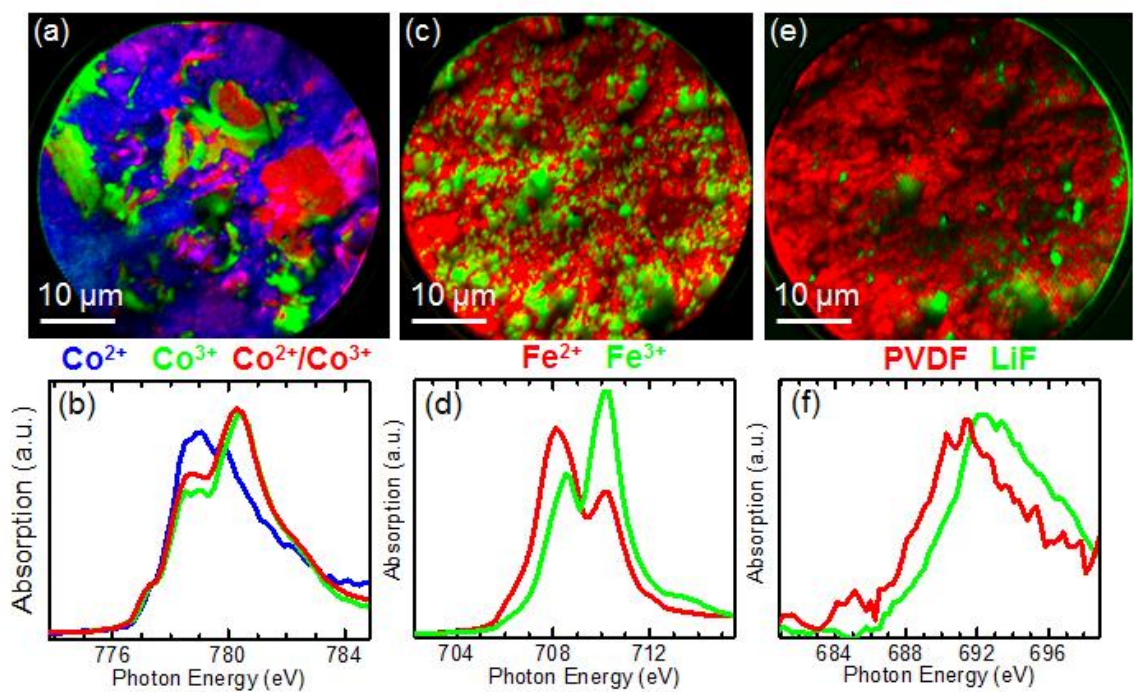

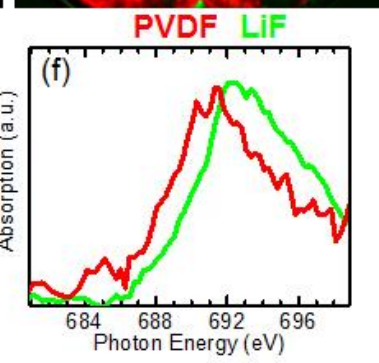

Figure 3. (a) X-PEEM chemical map, and (b) XANES at the Co Ledge of a $\mathrm{LiCoO}_{2}$ electrode surface cycled in $\mathrm{LiPF}_{6}$ with $\mathrm{SUN} / \mathrm{LiBOB}$ additives, blue: $\mathrm{Co}^{2+}$, green: $\mathrm{Co}^{3+}$, red: $\mathrm{Co}^{2+} / \mathrm{Co}^{3+}$ (adapted from [5]). (c) X-PEEM chemical map, and (d) XANES at the Fe L-edge of the state of charge on a cycled $\mathrm{LiFePO}_{4}$ battery surface, red: $\mathrm{Fe}^{2+}$, green: $\mathrm{Fe}^{3+}$; (e) X-PEEM chemical map, and (f) XANES at the F K-edge of the same surface as (c), red: PVDF, green: LiF (adapted from [6]).

on electrode morphology (crystal size, crystalline orientation), component surface chemistry, and local environments [5]. Three chemically distinct regions have been identified. The blue region refers to the electrode binder (PVDF) and carbon black (CB) materials. This region is fully covered by $\mathrm{Co}^{2+}$ species, $^{2}$ suggesting that the solid electrolyte interface (SEI) formed in this region. We concluded that the Co must come from LCO after dissolution by $\mathrm{LiPF}_{6}$ electrolyte. Therefore the $\mathrm{Co}^{2+}$ species is $\mathrm{CoF}_{2}$. The $\mathrm{LCO}$ regions (green for $\mathrm{LiCoO}_{2}$ and red for slight coexistence of $\mathrm{Co}^{2+}$ and $\mathrm{Co}^{3+}$ in $\mathrm{LCO}$ ) are also clearly identified. Some magenta colored regions show strong contributions of $\mathrm{CoF}_{2}$, suggesting $\mathrm{LCO}$ is partially covered by SEI. This is not seen without additives, implying the additive effect protects the electrode surface from further electrolyte corrosion. The electrode state of charge can also be readily imaged by XPEEM, as shown in Fig. 3c and 3d, which present chemical imaging and spectra of nanoscaled interfacial inhomogeneity in $\mathrm{LiFePO}_{4}$ (LFP) composite electrodes from a cycled large-format battery [6]. This particular electrode region, near the battery tab, shows an SOC corresponding to a mixture of $\mathrm{Li}_{0 .} \mathrm{FePO}_{4}$ and $\mathrm{FePO}_{4}$ and high Li loss. Regions away from the battery tab show quite different SOC and low Li loss. Finally SEI is identified as LiF in the cycled electrode (Fig. 3e and 3f), confirming the high stability of LFP in battery operation.

In summary, CLS STXMs and X-PEEM provide X-ray imaging and spectroscopy over a wide range of probing depths, transverse length scales and spatial resolutions. Soft X-ray spectromicroscopy has outstanding chemical sensitivity and site-specificity. It is well suited to lithium ion battery research [7].

\section{References:}

[1] K.V. Kaznatcheev et al, Nucl. Instr. Meth. Phys. Res. A 582 (2007) 96-99.

[2] J. Zhou, J. Wang et al, Chem. Commun. 49 (2013) 1765-1767.

[3] Xia Lu et al, J. Power Sources 329 (2016) 355-363.

[4] J. Zhou, J. Wang et al, Phys. Chem. Chem. Phys. 18 (2016) 22789-22793.

[5] J. Wang et al, Chem. Commun. 53 (2017) 8581-8584.

[6] J. Zhou, J. Wang et al, ACS Appl. Mater. Interfaces 9 (2017) 39336-39341.

[7] CLS is funded by CFI, NSERC, NRC, CIHR, the Government of Saskatchewan, WEDC, and the University of Saskatchewan. Research performed in collaboration with Dr. Jigang Zhou of CLS. Other collaborators are listed in the relevant publications. 
https://doi.org/10.1017/S1431927618014356 Published online by Cambridge University Press 